\title{
Interactive Visualization of 3D Histopathology in Native Resolution
}

Martin Falk, Anders Ynnerman, Darren Treanor and Claes Lundström

The self-archived postprint version of this journal article is available at Linköping University Institutional Repository (DiVA):

http:// urn.kb.se/ resolve?urn=urn:nbn:se:liu:diva- 150420

N.B.: When citing this work, cite the original publication.

Falk, M., Ynnerman, A., Treanor, D., Lundström, C., (2019), Interactive Visualization of 3D

Histopathology in Native Resolution, IEEE Transactions on Visualization and Computer Graphics, 25(1), . https:// doi.org/ 10.1109/ tvog.2018.2864816

Original publication available at:

https:// doi.org/ 10.1109/tvcg.2018.2864816

Copyright: Institute of Electrical and Electronics Engineers (IEEE)

http:// www.ieee.org/ index.html

(C)2019 IEEE. Personal use of this material is permitted. However, permission to reprint/republish this material for advertising or promotional purposes or for creating new collective works for resale or redistribution to servers or lists, or to reuse any copyrighted component of this work in other works must be obtained from the IEEE. 


\title{
Interactive Visualization of 3D Histopathology in Native Resolution
}

\author{
Martin Falk, Anders Ynnerman, Darren Treanor, and Claes Lundström
}

\begin{abstract}
We present a visualization application that enables effective interactive visual analysis of large-scale 3D histopathology, that is, high-resolution 3D microscopy data of human tissue. Clinical work flows and research based on pathology have, until now, largely been dominated by 2D imaging. As we will show in the paper, studying volumetric histology data will open up novel and useful opportunities for both research and clinical practice. Our starting point is the current lack of appropriate visualization tools in histopathology, which has been a limiting factor in the uptake of digital pathology. Visualization of 3D histology data does pose difficult challenges in several aspects. The full-color datasets are dense and large in scale, on the order of $100,000 \times 100,000 \times 100$ voxels This entails serious demands on both rendering performance and user experience design. Despite this, our developed application supports interactive study of 3D histology datasets at native resolution. Our application is based on tailoring and tuning of existing methods, system integration work, as well as a careful study of domain specific demands emanating from a close participatory design process with domain experts as team members. Results from a user evaluation employing the tool demonstrate a strong agreement among the 14 participating pathologists that 3D histopathology will be a valuable and enabling tool for their work.
\end{abstract}

Index Terms-Histology, Pathology, Volume Rendering, Expert Evaluation

\section{INTRODUCTION}

Pathology is the medical discipline studying the origin and diagnosis of disease at the cellular level. In health care, pathology assessments constitute decisive factors for a range of patient pathways, both regarding diagnosis and selection of treatment options. The examinations are made through expert analysis of imagery at the microscopic level of either coherent tissue (histopathology and cytopathology) or smears of detached cells (cytology). These analyses are also profound essential tools for medical research to better understand disease and to study treatment effects.

Histological samples are cut into thin sections, typically four micrometers thick, put on glass slides, and then stained with different stains for different purposes. The traditional tool in pathology for reviewing histological samples has thus been the light microscope. A modern option is to digitize the tissue image in a scanner and review the slide with dedicated computer software, which is known as WholeSlide Imaging (WSI). The WSI approach has been used in research settings for many years, but only recently started to make its way into clinical routine $[33,34]$. One reason for the late adoption of digital practices is the large size of the data; each slide can be on the order of $100,000 \times 100,000$ pixels.

Due to further advances in tissue sampling and image processing, it is now possible to create volumetric histopathology datasets. Much of the usefulness of these datasets has, however, remained out of reach since the visualization tools available have not been able to tackle the handling of this large and complex data. Even though 3D information has been available a reduction to 2D images has thus been the standard approach. Reducing dimensions, of course, brings limitations in terms of exploration possibilities, and it is likely that many opportunities for diagnostic understanding and biomedical research arise if the native 3D structure of tissue could be studied effectively.

- M. Falk and A. Ynnerman are with the Department of Science and Technology, Linköping University, Sweden.

E-mail: $\{$ martin.falk|anders.ynnerman\}@liu.se.

- D. Treanor is with the Leeds Teaching Hospitals NHS Trust, United Kingdom and the Center for Medical Image Science and Visualization (CMIV), Linköping University, Sweden. E-mail: darrentreanor@nhs.net.

- C. Lundström is with the Center for Medical Image Science and Visualization (CMIV), Linköping University, Sweden and Sectra AB. E-mail: claes.lundstrom@liu.se.

(C) 2018 IEEE. To appear in IEEE Transactions on Visualization and Computer Graphics Issue, 25(1), January 2019. For information on obtaining reprints of this article, please send a e-mail to: reprints@ieee.org. Digital Object Identifier: $10.1109 /$ TVCG.2018.2864816
To create a 3D histology dataset, the tissue sample is sectioned repeatedly and the resulting slides are scanned [29]. The 2D images are stacked and post-processed to form volumetric data. A typical dataset intended for research would be $100,000 \times 100,000 \times 100$ voxels. As will be detailed later in this paper, effective visualization of this data poses several challenges in terms of achieving both rendering pipeline performance and support for user experience of the complex interactions necessary.

In our work, we have addressed these challenges and, in collaboration with pathologists, developed a novel application for 3D histology visualization which opens up a new application domain. The main contributions of our work are:

- A visualization pipeline capable of handling 3D histology data in native resolution for interactive use.

- Domain-specific technical pipeline components and tailoring of methods, such as raycasting for RGB image stacks.

- User experience design including interaction methods for exploration of 3D histology data developed in a participatory design process with domain experts.

- A qualitative user evaluation employing our system, demonstrating the utility of 3D histology exploration.

\section{Related Work}

Two main areas of previous research efforts relevant to our work will be introduced below: digital pathology developments and certain volume rendering methods.

\subsection{Digital Pathology}

The digitization of microscopy slides has been done for many years for research and education purposes. In recent years, there has been a rapid development of digital pathology technology driven by the opportunity to introduce digital practices in routine clinical work $[33,34]$. The advances have included output quality and speed of scanners as well as software for data management and diagnostic review not only serving low-intensity research use but also high-intensity multi-user settings in clinical use [34].

Much research effort in digital pathology is focused on image analysis for diagnostic decision support, and the latest results clearly demonstrate that analytic algorithms can lead to improvements in efficiency and precision of diagnostic work $[3,32]$. Freely available frameworks like ImageJ [31] or 3D Slicer [1,12] provide functionality for image registration and segmentation applicable to digital pathology. SlideAtlas by Kitware, an open-source WSI platform, supports the alignment and browsing of multiple slide images [2]. 
3D histopathology is still a young area of research. The relevant previous work for this paper concerns datasets having appearance and resolution corresponding to conventional microscopy, being most relevant for clinical pathologists, whereas there are also other techniques producing 3D histology data [8,27,28]. Roberts et al. introduced a pipeline capable of producing 3D histology datasets from WSI scans [29] including registration to achieve a coherent volume. Many other efforts have been undertaken to address various challenges of 3D histology reconstruction such as multi-modal data [22] and particular applications [35]. The processing also commonly includes color normalization since staining quality typically differs between slides [19].

While the reconstruction of 3D histology data at native WSI resolution has been previously presented, the employed visualization systems have been limited to subsampled low-resolution and/or surfaceextracted versions and to basic interactions. Moreover, research on user interaction in histopathology has previously focused on WSI, i.e. to the 2D case. View navigation logs have been studied to reveal patterns and exploratory strategies $[24,30]$ and evaluations of different types of input devices have been carried out $[23,25]$.

\subsection{Volume Rendering}

In volume rendering, transfer functions play an important role in mapping data values to color and opacity $[9,21]$. In contrast to scalar data, dealing with volumetric color data, sometimes also referred to as photographic volumes, requires more elaborate transfer functions since colors must not be altered in order to retain the associated information while opacities need to be adjusted. This can be achieved by employing multidimensional transfer functions [20], gradient boundary enhancement based on colors [26], or utilizing color similarity [11]. The latter two approaches rely on the chromaticity of the CIE $\mathrm{L}^{*} \mathrm{u}^{*} \mathrm{v}$ color space for a more consistent user experience. To preserve the colors when performing the blending operation during raycasting, hue-preserving volume rendering based on chromatic compositing can be utilized [7].

Similar to 3D histology, cryogenic datasets such as the visible male cryosection data from the Visible Human Project also include color and can be of challenging data sizes which is why until a decade ago only smaller subsections were visualized, e.g. [10,26], or multi-resolution schemes are applied [13]. Another source for high-resolution data are electron microscopes where petascale volumes are obtained from a continuous stream of high-resolution electron microscopy images and subsequently being visualized utilizing brick-based rendering $[4,17]$. The data in this work is subdivided into bricks which are subsequently loaded into memory and then sampled during ray casting. For rendering, the bricks can be organized by different schemes like flat bricking, octrees [14], compressed hierarchical wavelet representations [16], or mixed-resolution approaches [5]. Typically, such schemes are also employed for culling empty bricks to improve the rendering performance. This is, however, not applicable in the context of histology since the datasets are dense and contain little to no empty space. For a detailed overview on GPU-based volume rendering of large-scale data we refer the interested reader to the work by Beyer et al. [6].

Brick-based raycasting is the backbone of our system for out-of-core rendering of 3D histology data on a regular desktop machine. We use a virtual volume for addressing the individual bricks [4]. In the approach by Beyer et al. [4] this corresponds to a region being imaged by an electron microscope and is used for detecting missing blocks during raycasting and dynamic level-of-detail computation. In our work, however, we deal with color data and the current view into the dataset, i.e. a subvolume, determines the required bricks and, thus, the virtual volume itself. Furthermore, we use a global level-of-detail based on the available image data and screen resolution. This results in fewer updates of the virtual volume and loading less bricks when adjusting the camera or panning the data.

\section{USAGE SCENARIOS}

This section presents the 3D histology visualization scenarios and their inherent design challenges. To provide an introduction to these we start by traditional WSI viewing in 2D, as parts of the visualization and interaction take its starting point there.

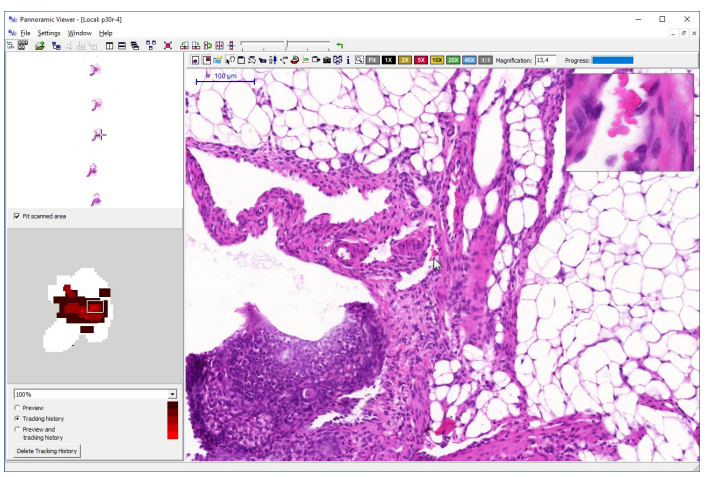

Fig. 1. Example of a whole-slide imaging tool for 2D histopathology (Pannoramic Viewer by 3DHISTECH Ltd.). The main view (center) shows the tissue sample at arbitrary magnifications while a minimap indicating the current position is located on the left. An additional heatmap indicates visited areas. The most prevalent interactions involve panning and zooming.

\subsection{Reference: WSI/2D Histopathology}

The digital part of WSI begins when the glass slide with the prepared and stained tissue sample is scanned. Usually the scanning resolution is $0.25 \mu \mathrm{m}$ per pixel which is intended to correspond to the view in a microscope at 400 times magnification. As a standard glass slide is about $75 \mathrm{~mm}$ by $25 \mathrm{~mm}$ in size, the resulting color image can exceed 100,000 pixels in both dimensions.

Once the pathologist obtains access to the image file, she can begin with the clinical diagnosis. The image review consists of exploring each slide at different zoom levels in the search for malignancies. Structures, cell types, and cell states (such as mitosis) are examples of findings that lead to diagnostic conclusions on cancer or other conditions. In some cases, the pathologist is required to produce a quantitative measure such as the percentage of positively stained cells in a hot spot region.

The tools used are quite similar and typically feature a large central view of the specimen, i.e. the digitized image. Fig. 1 depicts such an application. The main view is most often complemented with a minimap or thumbnail. This minimap provides an overview while pointing out the current location at the same time, and can be used for navigation purposes. Information on which regions the pathologist has displayed in the main view can be overlayed as a heatmap on the minimap.

The interactions extensively performed in these kinds of tools are panning and zooming [24] controlled by a mouse or other input devices [25]. Panning enables the user to explore the extent of the image. The appropriate zoom level heavily depends on the diagnostic task at hand and it is often necessary to quickly move between overview and detail.

\subsection{D Histopathology}

Taking a 2D section from a tissue specimen is a sampling that corresponds to an extreme reduction of the available information. For example in a typical, $5 \mathrm{~mm}$ thick tissue sample, often only a single $5 \mu \mathrm{m}$ section, i.e. a $1 / 1000^{\text {th }}$ of the volume, is examined. To mitigate the risk for missing important findings, clinical protocols often require a few sections being made at several levels. The distance in between levels is in these cases, however, relatively large - many tens or hundreds of intermediate sections are discarded. By 3D histopathology we instead refer to datasets built from consecutive or being close to consecutive sections.

In our work, we have used 3D histopathology datasets with more than a hundred sections [29]. Each slide is prepared as in the regular 2D work flow. After scanning with a Leica Aperio AT2 scanner the WSI images are joined into a slide stack. Extensive post-processing is, however, needed to turn it into a coherent volumetric dataset. Registration of slices is required since the preparation affects the non-rigid tissue sections differently [22]. It might also be necessary to apply color normalization to adjust for staining differences. Fig. 2 shows an example 

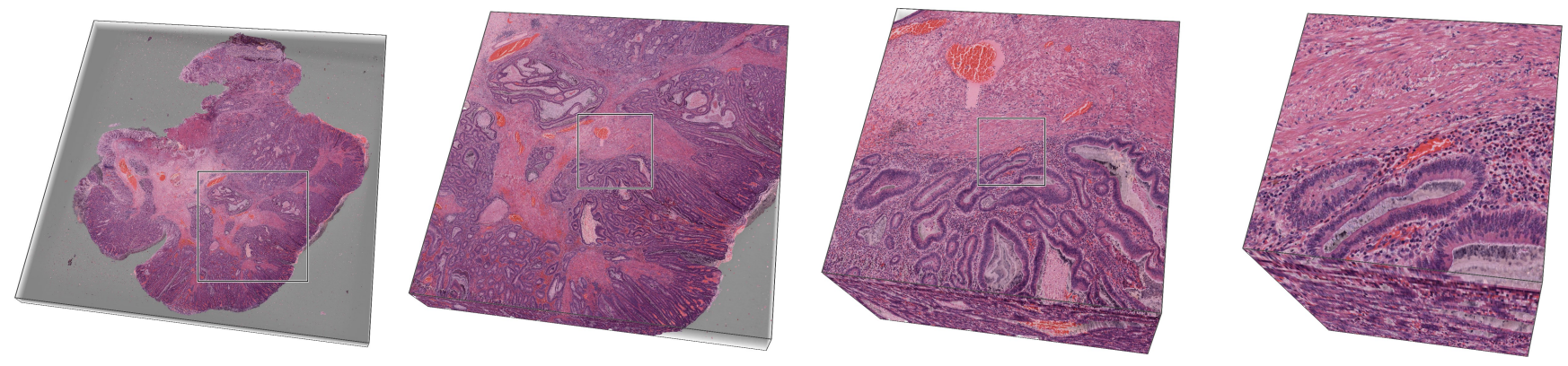

Fig. 2. Slide stack of a colorectal adenocarcinoma consisting of 50 slide images scanned at 20x. The length of the bounding box measures $22 \mathrm{~mm}$, $8 \mathrm{~mm}, 2 \mathrm{~mm}$, and $500 \mu \mathrm{m}$ from left to right. Thus, one pixel corresponds to $23 \mu \mathrm{m}, 4 \mu \mathrm{m}, 2 \mu \mathrm{m}$, and $0.5 \mu \mathrm{m}$, respectively. The highlighted region indicates the approximate location of the subsequent visualization to the right. Individual cell nuclei are discernible as dark purple points.

of a colorectal adenocarcinoma slide stack at different magnifications with length scales ranging from $22 \mathrm{~mm}$ down to $500 \mu \mathrm{m}$.

A main objective of this work is to investigate if a high-performing visualization system opens possibilities for use of 3D histology both in research and clinical settings. In research this would be an extension of the utility achieved by the basic low-resolution visualizations now available whereas this would be a true novelty in clinical work. In current practice, i.e. 2D imaging or at best just a few slides at different levels of the specimen, the pathologist must rely on building a mental model of the three-dimensional structures. In our discussions with domain experts they underline that the spatial 3D tissue model in their mind is often coarse and limited. There are many situations where a more detailed volumetric understanding of the tissue constitution could make a difference. For instance, the impact or relevance of elongated structures such as vessels and crypts are difficult to assess from a single slice. Moreover, fully understanding the shape and spatial relations of tumors, glands, polyps, and other tissue components can have an impact on diagnostic conclusions. It should be noted that the extensive work required for preparing datasets prohibits 3D histology from being commonly applied in clinical practice at the present time. But once the utility is realized in practice there is a strong incentive to also streamline the preparation process.

Visualizing 3D histology entails a number of challenges beyond what traditionally has been used in WSI and other medical 3D rendering. The main demands that our visualization system needed to address were as follows:

- Handling of the extreme data sizes, on the order of $100,000 \times$ $100,000 \times 100$ voxels, with frequent zoom interactions from low to high magnification and back.

- Handling of highly non-isotropic voxels.

- Preservation of native tissue staining colors, as they have a distinct diagnostic meaning for the pathologist.

- Separated rendering of the background, being natively opaque white in the data and thereby otherwise representing empty regions incorrectly in 3D.

- Providing orientation and position context even in close-up views where the dense data has no orientation-revealing features.

- Providing arbitrary clipping without causing additional orientation difficulties in close-up views.

- Providing means for all needed manipulation without overwhelming users with too many interactions.

In the following, we describe how we address these challenges from a visualization point of view.

\section{System Design}

We will in this section describe the developed 3D histopathology system. After briefly describing the design process, we present an overview of the user interface followed by the design of the connected viewing modes and user interactions, and finally we provide the technical details of the rendering pipeline.

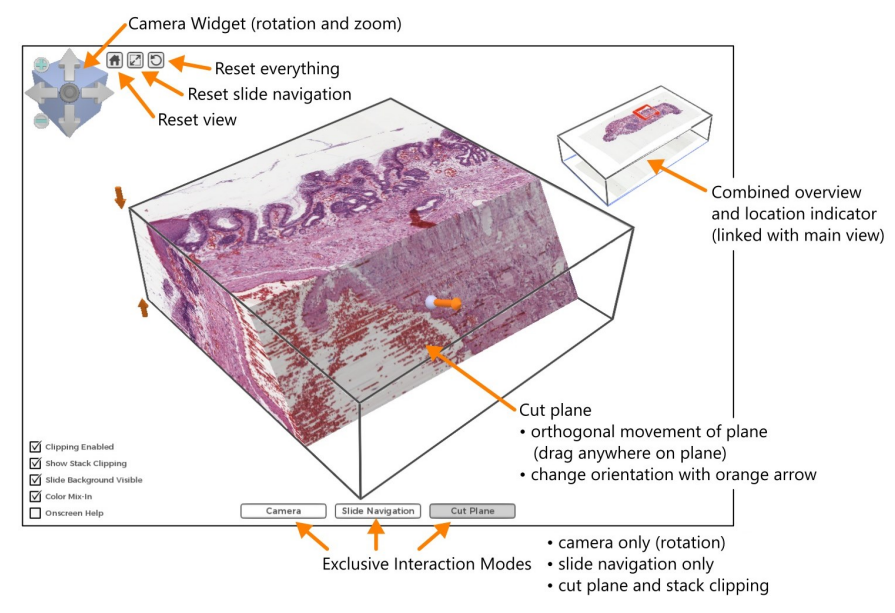

Fig. 3. Overview depicting the different interaction elements of our tool.

\subsection{Design Process}

The software was developed in a participatory design process with the domain experts, i.e. pathologists working in a clinical environment. Pathology expertise was available both in the cross-disciplinary research team itself and through interaction with clinical partners. The software development was conducted in iterative fashion with regular meetings where the system was demonstrated to and operated by pathologists to identify weak points early on.

Once the software reached a mature state with a viable set of features we performed a pilot study. In total, three persons participated; one pathologist and two clinical research staff at a hospital in Sweden. All participants were familiar with WSI software and had experience in histopathology.

On the one hand, the purpose of the pilot study was to try out the study design as a predecessor to the main evaluation. Thus, the pilot to a large extent followed the setup discussed in Section 5. The changes made after the pilot included slight tuning of the task descriptions and extension of up-front information given to the participants. The second purpose of the pilot was to get thorough feedback on the end-to-end system which led to a final round of improvements to the system.

\subsection{User Interface Overview}

The final version of the user interface is shown in Fig. 3. The user interface is contained within a single window to avoid having the user switch between multiple contexts. The main view of the dataset is presented in the center. There is a widget for camera control (top left) and some basic options are controlled by check boxes (bottom left).

An effective visualization of this type of data needs to deal with the issue that users may easily lose their orientation in the data. This becomes pertinent as soon as the magnification is increased such that less than the full dataset fits into the viewport. The density of the 

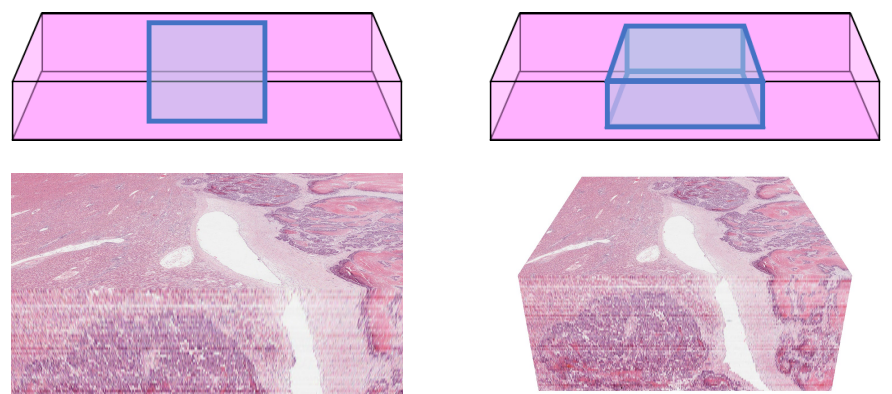

(a)

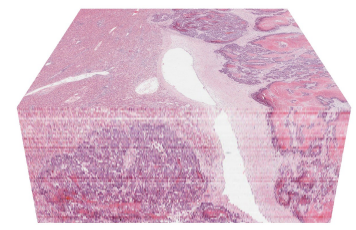

(b)

Fig. 4. Comparing the global view of the slide stack being cropped by the viewport (a) with a subvolume view (b). The global slide stack is clipped in the lower third but the orientation of the clip plane is not apparent. The same excerpt is easier to relate to spatially when represented as subvolume thanks to the boundaries and the principle of closure.

data means that volumetric understanding from objects at depth is not possible as such parts are typically obscured. Moreover, in contrast to radiology imaging, there are no features with known orientation that can provide spatial cues. Several design choices were made to address this challenge. A minimap is always shown in the top right corner, providing an overview of the full dataset and its orientation linked with the main view. The subvolume seen in the main view is also marked in the minimap. Clipping from the top, for the user typically interpreted as browsing of the slide stack, is represented in the minimap by the slide shown. The main view shows a bounding box, always depicting the three original axes, which assists understanding of orientation in particular for oblique clipping planes. Other features to assist spatial control are the options to revert both view and slide stack parameters to the original state, i.e. resetting the orientation of the volume and showing the entire dataset, available as buttons next to the camera widget.

In order to reduce the overall complexity of available interaction at hand, we introduced distinct interaction modes selectable via buttons at the bottom. The three simple modes limit the interaction to slide navigation, camera manipulation, and positioning the clip plane, respectively.

The three modes can only be used one at a time. While the limited modes are intended for novice users, there is also an expert mode with all interactions available simultaneously for pathologists having gained more experience with the system. In expert mode, mouse interactions on top of the stack are mapped to slide navigation, i.e. panning and zoom, while interactions at other positions in the window control the camera. When pressing a modifier key the clip plane is adjusted with the mouse.

\subsection{View and Interaction Design}

In 3D histopathology, a scene extends over a couple of centimeters, with details being available down to sub-micrometer level. A fundamental piece of the visualization system development was to design the scene composition for which two approaches have been considered. The straightforward way would be to create a global scene containing the stack of slide images. In this approach, the selection of which parts of the data are visible, and at what level of detail, is determined by camera navigation alone. Moving the camera close to the slide stack reduces the visible region and increases the level of detail. The second approach is to use a subvolume revealing only a small part of the dataset at a time The subvolume selection, i.e. the data window, is in this case handled explicitly by interactions separate from camera movements.

From a simplicity perspective, the fact that the set of user interactions is larger in the subvolume approach should be a considerable disadvantage. However, the challenge of providing orientation cues at high magnification, described in the previous section, turned out to be the most important aspect for which to find an effective solution.

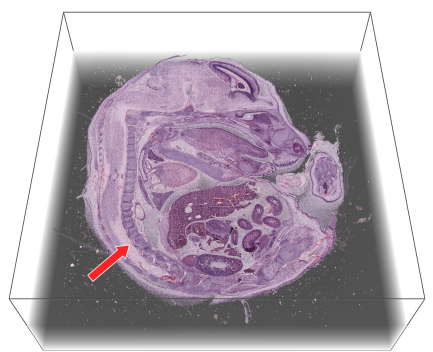

(a)

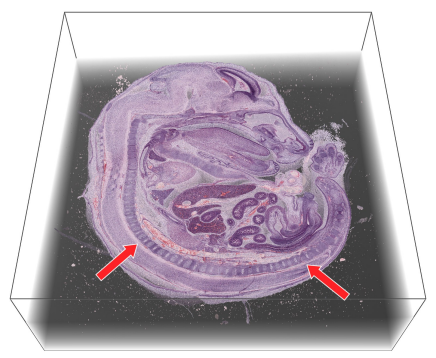

(b)
Fig. 5. Revealing the spinal column of the mouse embryo dataset by cropping the slide stack (a) and using a custom clip plane (b).

In fact, the global scene approach fails to provide a proper spatial context in close-up views. This effect is illustrated in Fig. 4, where the global scene approach (left) is compared to the subvolume approach (right) for the same data. The dense and homogeneous data excerpt does not provide cues for the spatial constitution. The transition in the global scene between top and sides of the volume is hardly visible and the orientation of the clip plane is not apparent at all. For the subvolume, however, the principle of closure, i.e. the tendency of the mind to complete forms, provides more contextual information. The shape of the subvolume, i.e. a cuboid, is completed and, thus, the edges are more pronounced. Another advantage of the subvolume approach is that investigating cross-sections is readily available by panning the tissue, effectively moving subvolume boundaries. A similar effect could also be achieved by using four additional clip planes in the global scene which, however, comes at additional costs for both interaction and data handling.

The user feedback clearly showed that the subvolume approach is the best option for this application, and we therefore adopted it in our system. This conclusion is an interesting and perhaps counter-intuitive result that may have bearing in other visualization domains:

In terms of visualization user experience, the advantage of higher perceptual simplicity may outweigh the burden of a larger set of interactions.

Interacting with a slide stack in three dimensions offers a higher degree of freedom compared to the inspection of a regular, small-scale volume or when exploring a single high-resolution image. With the subvolume approach the interactions include the two categories of camera manipulation and data navigation. A regular camera is used for setting the view parameters including rotation, dolly, and tilt. In order to maintain the contextual information provided by the subvolume, i.e. its cuboid shape and extent, the camera dolly is restricted such that the borders of the subvolume are visible at all times.

Data navigation consists of panning, zooming, and vertical stack browsing. Both panning and zooming are used to determine the contents depicted in the subvolume mimicking the interactions used in WSI (cf. Section 3.1). Since the number of slices within a stack is rather low compared to the horizontal extent, panning is restricted to the horizontal plane and does not affect the number of visible stack slices. Zooming in the horizontal plane is a straightforward increase of the level of detail. With the viewport fixed, zooming also decreases the spatial extent of the subvolume. To achieve a zooming effect in the vertical plane, the spacing between the slides is increased to preserve physical proportions. Stack browsing refers to interactively clipping the stack along its vertical axis from either side to reveal individual slide images. This enables pathologists to inspect the raw data of each individual slide image similar to WSI.

Showing axis-aligned cross sections is performed through the subvolume panning described above. The targeted use cases also include clipping in arbitrary oblique planes. One reason is that the orientation of the specimen is undefined, and anatomically relevant directions are typically not aligned with the main dataset axes. A custom clip plane 


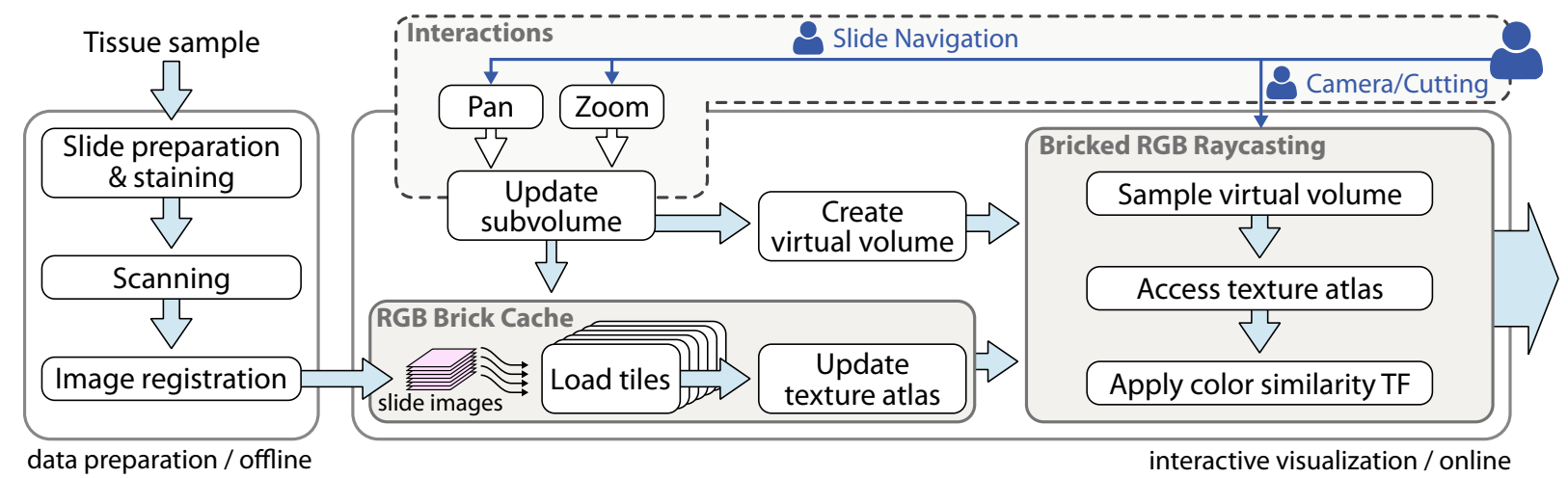

Fig. 6. Our rendering pipeline for the interactive visualization of 3D histopathology data at native resolution.

allows for inspecting features where the region or plane of interest might not be aligned with the slide stack, e.g. the spinal column of a mouse embryo (Fig. 5) or glandular structures (Fig. 13). The clip plane is constrained to the inside of the subvolume and can be moved along its normal direction. An arrow located at the plane's center of mass indicates the plane normal (Fig. 3) while dragging the arrow changes the plane orientation.

Another important requirement for 3D histology relates to the fact that most tissue is dense with little to no empty space within. To see beyond opaque block faces and appreciate the 3D appearance of structures of interest, the ability to lower the visibility of less relevant regions is needed. The most prominent example of this is the removal of the white "slide background" of the scanned glass slides, the area outside the actual specimen and inside cavities.

We found that the transfer function method based on color similarity [11] addresses this issue well by using only a single transfer function widget targeted toward white color. In order to limit the interaction complexity we provide the user with two transfer function presets: one preset assigning a low opacity to white areas, thereby suppressing the slide background, and the second one showing the unmodified, original data. The user can switch between these two presets by means of a check box (Fig. 3). To further emphasize variation in depth and reduce visual clutter, we provide the option to replace the slide background with a semitransparent black, see Fig. 5 and 8. This is also useful in subvolume close-ups as illustrated by the accentuated spatial structures in Fig. 8. At the detailed level, the datasets can be noisy due to scanning artifacts and occurrence of small debris of tissue. A sharp transition in the visibility transfer function typically results in unwanted accentuation of noisy details. Therefore, a gradual opacity fall-off is employed in our visualizations.

\subsection{Rendering Pipeline}

Fig. 6 depicts the rendering pipeline of our visualization system. In order to create a slide stack, we perform an image-based registration according to Magee et al. [22] in the data preparation stage. We can either store only the required transformations and reconstruct the registration on-the-fly while loading the data, or apply the image registration and export new slide images. The former has the advantage of working with the original data instead of an intermediate representation. This, however, comes at the cost of additional overhead when loading the image data since larger areas have to be sampled in order to cover spatially adjusted regions. To reduce the sampling time when accessing the image data we therefore opted for the second approach. In contrast to Magee et al. we store individual high-resolution images rather than precomputed, low-resolution volumes on disk.

Many formats for digital slide images implement some kind of tiling strategy for efficient data handling. One example is the pyramidal TIFF format featuring a mipmap hierarchy besides image tiles making it perfectly suitable for out-of-core data access and rendering, a fact we utilize in our system. Please note that such a mipmap hierarchy must not necessarily be complete, i.e. levels might be missing, and hence the need arises to reconstruct those levels either during pre-processing or while loading the image data. We use the OpenSlide image library [15] for accessing this data. The library supports accessing subregions and a wide variety of formats for microscope slide images.

In the visualization stage, we employ brick-based volume raycasting [6] to render the slide stack, using the image tiles to construct the brick structure. The key differences to regular brick-based rendering are that we are dealing with color data and that the height of the stack, i.e. the number of slide images, remains the same at all times independent of the level of detail.

For rendering, the visible bricks are determined by the current viewport, the magnification level, and the extent of the subvolume visible on screen. Slide navigation and zooming directly affect the subvolume (cf. Section 4.3) and, thus, data selection. Each time the subvolume is updated, the required bricks are requested from the brick cache which triggers the loading of missing bricks. For each missing brick, the corresponding image tiles are first loaded using the OpenSlide library before being cached in a 3D texture atlas on the GPU. To avoid interpolation artifacts in between bricks, voxels located at brick boundaries can be duplicated in the texture atlas [18].

We can then generate a virtual volume by combining the subvolume and already existing bricks. The virtual volume represents individual bricks by one voxel each and is used for indirection during raycasting [4]. It is a uniform grid implicitly supporting multi-resolution rendering, i.e. bricks of lower resolution cover more than one voxel. Each brick voxel stores an atlas ID (if there are multiple atlases), the indexed location within the atlas, a scaling factor, and the local offset of the current brick. Offsets and scaling factors are necessary to correctly sample and scale low resolution bricks.

The virtual volume is then passed on to an RGB raycasting stage along with the texture atlas. For opacity mapping we utilize a 2D transfer function based on color similarity [11]. During raycasting, all color samples are transformed into a perception-based color space to compute a color similarity based on the distance between the transformed color and a transfer function primitive which is then mapped to opacity. Optionally, replacement colors can be defined, which could e.g. be used for highlighting features.

When applying trilinear interpolation during raycasting, the sides of the slide stack appear blurry due to the low vertical resolution. By modifying the texture access along the vertical axis we can emphasize the individual slides and reduce the blur. Let $z$ be the vertical component of the texture coordinate in the range $[0, n]$, then the modified, nonlinear coordinate $z^{\prime}$ is computed as follows

$$
\begin{aligned}
& a=2 \operatorname{frac}(z)-1, \quad \operatorname{frac}(x)= \begin{cases}x-\lfloor x\rfloor & x \geq 0 \\
x-\lceil x\rceil & x<0\end{cases} \\
& z^{\prime}=\lfloor z\rfloor+\frac{\operatorname{sign}(a)|a|^{\lambda}+1}{2} .
\end{aligned}
$$

With $\lambda=1$ this is equivalent to linear interpolation while $\lambda \rightarrow \infty$ reflects nearest neighbor interpolation. This adjustment is easily integrated in 

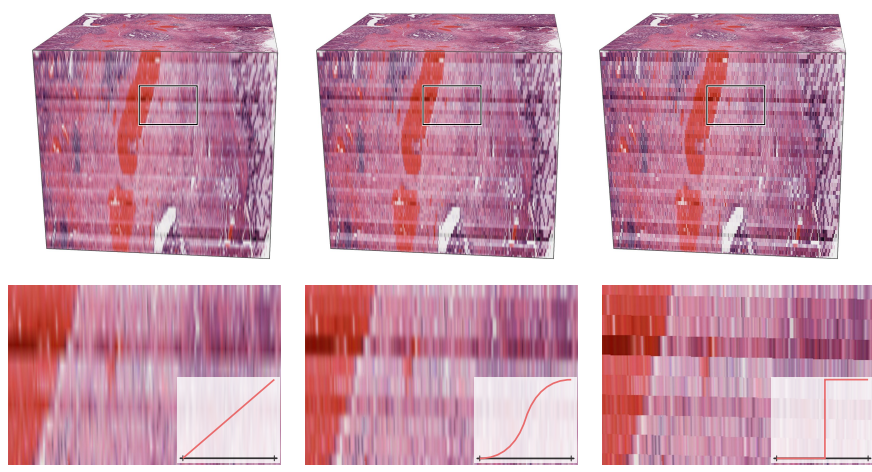

Fig. 7. Emphasizing individual slide images inside the stack by modifying the vertical interpolation in the raycasting step: regular linear interpolation, nonlinear interpolation with $\lambda=3$, and nearest neighbor (left to right). Data: colorectal adenocarcinoma, H\&E stain.

the raycasting stage by modifying the vertical component of the current ray position before accessing the atlas texture with regular trilinear interpolation. Fig. 7 depicts the difference between vertical linear and nonlinear interpolation for $\lambda=3$ as well as the nearest slide image. Using nonlinear interpolation, the individual slide images are more distinct while providing smooth transitions between them at the same time.

The core functionality of our system is implemented in the Inviwo visualization framework (www. inviwo.org) using C++ and OpenGL. The framework provides a thread pool, which is used for on-demand asynchronous loading of bricked image data in the background, thereby decoupling user interaction and volume rendering from data loading.

\section{Qualitative User Evaluation}

We performed a domain-expert user evaluation at two university hospitals, one in Sweden and one in England. Our study was the first exposure to 3D histology exploration for most of the participants, and such tasks have no existing alternative solution in current practice. The distinct novelty of this application, having no comparable baseline, entails that quantitative measures of task efficiency and accuracy would have limited value, even if such evaluations normally are of great interest in visualization research. Instead, we opted for a qualitative evaluation with the primary aim to investigate whether 3D histology visualization would be beneficial for clinical and research use cases in pathology. In addition, all feedback and observations during the study were gathered in order to inform further development of our system.

\subsection{Evaluation Setup}

The evaluation was laid out as a guided think-aloud study, i.e. the participants were encouraged to communicate their thoughts and concerns. From the pilot study we noticed that with the limited training time available, participants sometimes lost the spatial orientation in the 3D data domain. To amend this, we included a guidance possibility by having one of the authors present at all times to intervene and help when necessary.

The individual sessions were scheduled to take between 45 to 60 minutes and there was no remuneration. After a brief introduction on the aims of the study, the participants were handed a questionnaire and written instructions including brief descriptions of the datasets. The questionnaire had two parts, the first covering background questions and the second for reporting feedback on the visualization, filled out after performing the tasks.

Task 1 consisted of an exploration of a Barrett's esophagus dataset (Fig. 9a), chosen as an appropriate introductory case due to its distinct spatial characteristics. Apart from investigating the value of 3D exploration in this diagnostic area, the task was intended to familiarize the participant with the tool and its capabilities. The illustration shown in Fig. 3 was given in the task description as a reference. The participants were given 10 minutes to freely explore the dataset and experiment

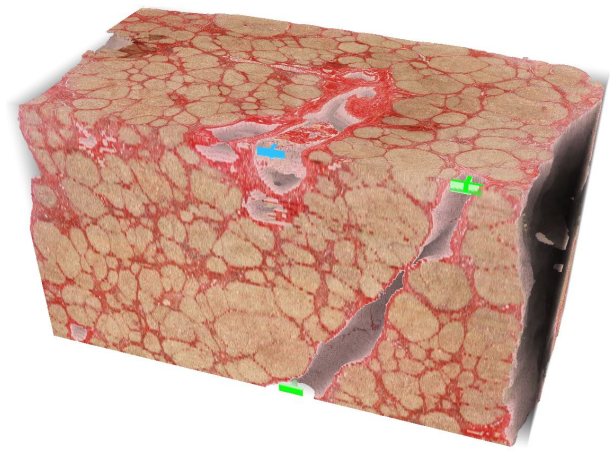

Fig. 8. Liver cirrhosis dataset with a Sirius Red stain used in Task 2 of the evaluation. The task included the identification of pairs of colored markers which are connected through a vessel.

with navigation controls, stack browsing, clipping, and background removal.

Task 2 consisted of a specific case where 3D exploration was particularly interesting to study: the tracking of structures like vessels or glands. For this purpose, we enriched the liver cirrhosis dataset by adding small colored 3D markers in the shape of a plus sign to various vessels. The task was to identify pairs of markers which were connected through the same vessel. Fig. 8 depicts such a connected marker pair revealed by applying a clip plane. Note that the Sirius Red staining used in this dataset leaves the arteries white, and thanks to the color similarity transfer function they become transparent. During this task, the slide navigation including panning and zoom was disabled in order to focus on camera interaction, stack browsing, and clipping.

As we anticipated that the pathologists would give the richest feedback for datasets relating to their personal interest and expertise, we designed Task 3 as an open-ended exploration. The participant could choose among a number of datasets (see Sec. 5.2) as they wished, and they freely decided their own exploratory agenda. The task design particularly targeted understanding the medical insights the participants could elicit from the visualization without priming the participants with preconceived notions of benefits.

\subsection{Datasets}

We provided a wide selection of datasets covering both medical cases pathologists are dealing with on a daily basis as well as unfamiliar examples intended to spark discussions. The available datasets are depicted in Fig. 9. The noise surrounding the specimen originates from tissue debris on the microscope slide and in case of the carcinoma also from compression artifacts.

Barrett's esophagus (Task 1): This is a medical condition in the lower esophagus, potentially resulting in cancer if not treated. Here, the stomach acid causes abnormal cell changes, visible as a distinct alteration in the appearance of the tissue from normal lining ("squamous epithelium") to abnormal ("glandular epithelium"). The abnormal lining has a complex 3D architecture compared to a simple flat 2D architecture in normal tissue. The 102 microscope slides are stained with H\&E (haematoxylin and eosin) and were scanned at 40x. Each image has a resolution of $64,000 \times 35,000$ pixels corresponding to $16 \mathrm{~mm}$ by $9 \mathrm{~mm}$.

Liver cirrhosis (Task 2): The tissue changes in cirrhosis comprise an increase in fibrous (scar) tissue in the liver, resulting in a complex 3D scar formation with "nodules" of liver tissue surrounded by fibrous scars. The Sirius red stain marks cells in yellow-orange, collagen in red, and highlights the scar tissue of the cirrhosis in bright red while blood vessels remain white. The goal of Task 2 is to locate and follow vessels extending over several millimeters which are clearly visible when looking at the entire dataset at low magnification. Thus, a downsampled dataset is sufficient for this task and does not affect the significance since the task does not require close-up views. Employing a downsampled version $(1,250 \times 1,114$ pixels $)$ also has a very practical reason, 


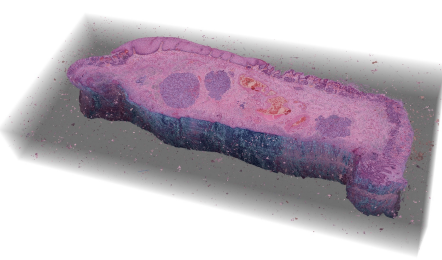

(a)

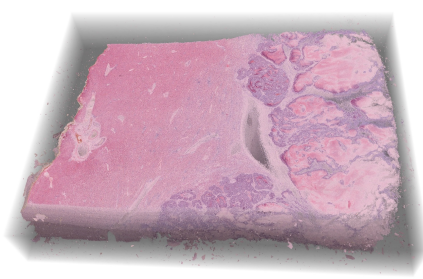

(b)

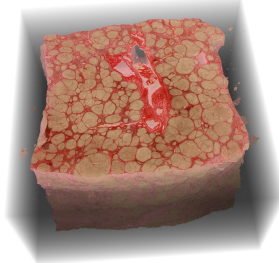

(c)

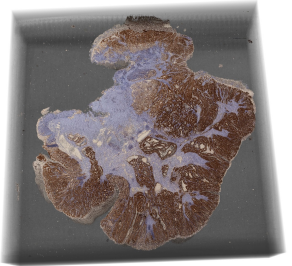

(d)

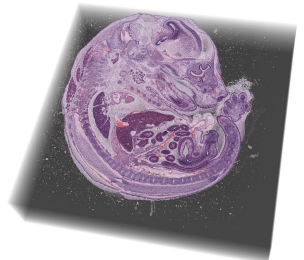

(e)

Fig. 9. Datasets available for inspection in the open-ended exploration task of the expert evaluation. (a) Barrett's esophagus, H\&E stain. (b) healthy liver, H\&E stain. (c) liver cirrhosis, Sirius red stain. (d) colorectal adenocarcinoma, immunostaining. (e) mouse embryo, H\&E stain.

i.e. the embedding of the additional color markers with minimal effort (Fig. 8). The full-resolution slide images, digitized at 20x, measure $53,000 \times 48,000$ pixels or $27 \mathrm{~mm}$ by $24 \mathrm{~mm}$ with 96 images in total.

Healthy liver: This dataset was chosen since many of the candidates participating in our evaluation are experienced in gastrointestinal pathology. It contains 70 slices of a healthy liver $7 \mu \mathrm{m}$ apart, each scanned at $20 x$ resulting in $63,000 \times 45,000$ pixels or $32 \mathrm{~mm}$ by $23 \mathrm{~mm}$.

Colorectal adenocarcinoma: The data depicts the early stage of a carcinoma arising in a colorectal polyp (Fig. 2). Here, the datasets contains 50 slide images with an interslice distance of $20 \mu \mathrm{m}$ digitized twice at 20x, once with an H\&E stain and a second time with a keratin immunostain thereby revealing the epithelium in brown. The image resolution is $56,000 \times 56,000$ pixels or $28 \mathrm{~mm}$ by $28 \mathrm{~mm}$.

Mouse embryo: The fully developed embryo of a mouse is captured in a curled up pose. Using an H\&E stain reveals distinct features like limbs, eyes, organs, and hair follicles on the snout. The specimen measures $14 \mathrm{~mm}$ by $13 \mathrm{~mm}$ by $6 \mathrm{~mm}$ and, when scanned at 40x, resulted in a volume of $55,000 \times 53,000 \times 110$ voxels.

\subsection{Participants}

We had 14 pathologists participating, eight from hospital A in Sweden and six from hospital B in England. Their age was between 27 and 62 years. All were practicing hospital pathologists, but many also conduct research to some degree (see Fig. 10). Subspecialties represented among the pathologists were varying: gastrointestinal (8), molecular (2), soft tissue (2), uro (2), gynecological (1), renal (1), breast (1), neuro (1), and perinatal/prenatal (2) pathology, as well as cytology (2). Three of the participants were pathologist residents, but the somewhat limited experience did not cause problems to understand the medical context of the datasets or to carry out the tasks.

One aspect of the pathologists' experience with potential bearing on the 3D histology evaluation is their experience of WSI, i.e. digital imaging in 2D. As seen in Fig. 11, frequent microscope analysis was reported, whereas the usage of digital slides was somewhat less frequent. The bottom graph of Fig. 11 shows that the pathologists working digitally more often also have a tendency to favor WSI over the microscope.

\section{Results \& Discussion}

The evaluation results are presented with respect to several aspects: explicit feedback on utility, other observations during the tasks, medical insights gleaned, and technical performance.

\subsection{Utility Feedback}

After the interactive tasks, the participants answered questions regarding their experiences and considerations. The questions were posed in form of a 6-point Likert scale and were formulated as follows:

- Does the 3D visualization support you in building a spatial, mental model of the tissue?

- Is it easier for you to study spatial structures in 3D than in 2D?

- Would you consider the 3D visualization to be helpful in your current work?

- Would you use it in your daily workflow regarding research if it was available?

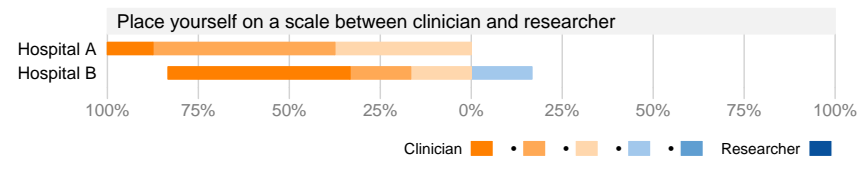

Fig. 10. Responses of 14 experts located at two hospitals regarding whether they see themselves more on the clinical side or the research side ( 8 from hospital A and 6 from hospital B). The length of each bar represents the percentage of people choosing this particular response.

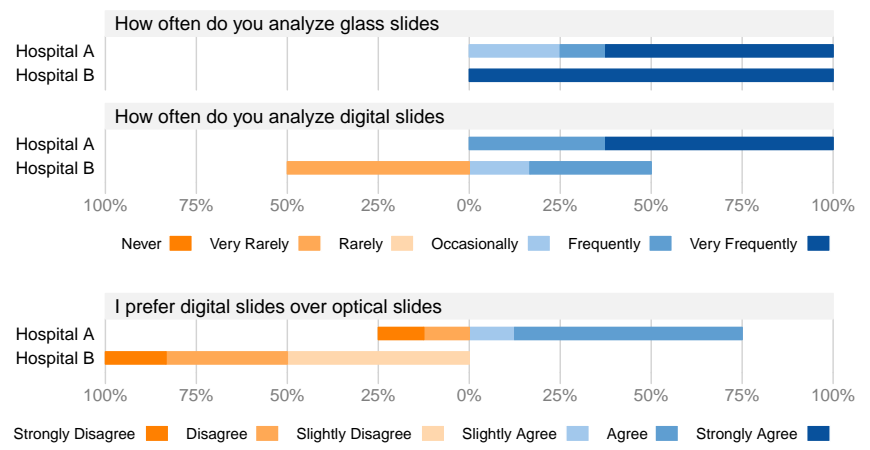

Fig. 11. Comparing the usage frequency and preference for microscope glass slides and digital slide images ( 8 from hospital $A$ and 6 from hospital $\mathrm{B})$. The length of each bar represents the percentage of people choosing this particular response.

- Can you see the 3D visualization being applied in your daily clinical work?

- How would you rate the perceived usefulness of the tool when solving the tasks?

The questionnaire was concluded by a free text question regarding insights gained during the exploration. The aggregated responses are depicted in Fig. 12.

All of the participants agree that a 3D tissue representation aids them in building a mental model of the sample. Moreover, the pathologists also considered it easier to study those structures in our 3D setup compared to browsing through $2 \mathrm{D}$ images, which is reflected by the positive responses to the second question. Participant comments further emphasized the value of having a correct and direct 3D representation, as when referring to the 2D practice as follows: "Pathologists think in three dimensions where the image is created in your brain based on guess work." Comments were made that the slide stack visualization "clearly fills a gap" in histology understanding and showed "must-have potential." Furthermore, the darkening of transparent regions helped to "really understand cavities", and the additional third dimension was considered to have some impact on the precision of measurement tasks.

As seen in Fig. 12, the general consensus was that visualizing slide stacks in 3D would be helpful in some but not all cases. In particular, there seems to be no necessity for applying it to every case, e.g. in the 

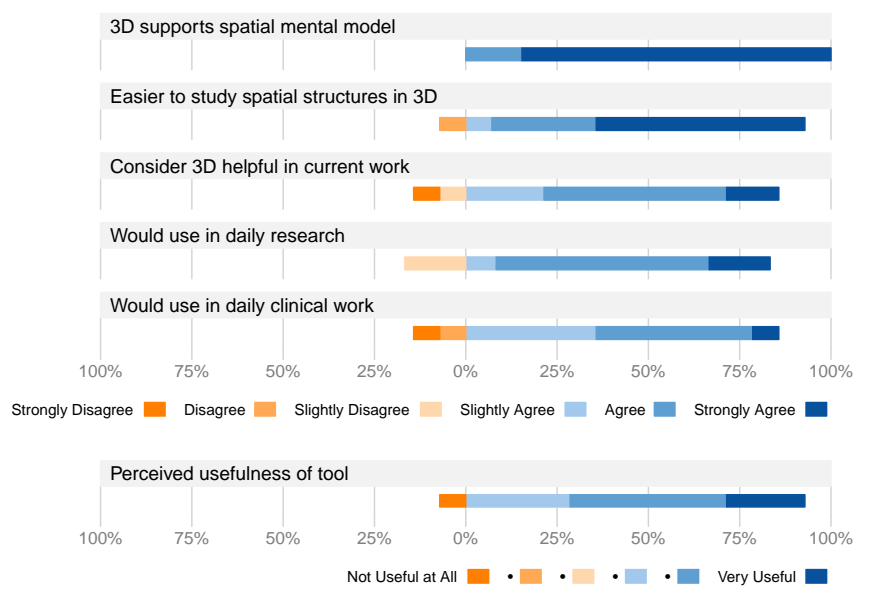

Fig. 12. Responses of our expert evaluation. The length of each bar represents the percentage of people choosing this particular response.

case of the healthy liver where one or two slide images are sufficient for clinical diagnosis.

The outliers with negative responses correspond to two specific participants, one referring to not seeing potential use cases in his own work and the other bringing up higher costs of dataset preparation.

We could not find a correlation between preferring digital slides over the microscope (cf. Section 5.3) and agreeing more with the utility of 3D. There was also no noticeable difference between pathologist residents and more experienced pathologists.

\subsection{Observations During Tasks}

Much of the obtained feedback was attributed to the 3D histology visualization in general. It was the first time working with an interactive slide stack for most of the participants. Therefore, many comments were quite generic and included phrases like "this is amazing" and " $I$ am fascinated." On the negative side, people were concerned with the fact that it is "tricky to grasp so much information" and that one might "spend an awful lot of time on beautiful images."

The actions taken by each participant for solving the tasks were quite similar. Some initial difficulties when interacting with the 3D camera were common. Nevertheless, most of the time was spent manipulating the camera to look at the data from different angles. Slide navigation, i.e. zooming and panning, was utilized less often and mostly only for occasionally closing in on interesting features while retaining some context. Overall, most participants were very active in their exploration, whereas a few were more passive and often asked for hands-on guidance.

An interesting observation is that stack browsing was used less and less in favor of custom clipping over the course of each experiment. The custom clipping was particularly useful in Task 2 where many participants first found one marker and then adjusted the clip plane in order to reveal the cross section of the vessel and find a matching marker (see Fig. 8). A more 2D-based approach was also used by some participants: tracking the vessel while cropping the stack until the second marker was found.

The observations of the use of 2D-centric vs. 3D-centric interaction constitute a finding that can be valuable to apply also in other visualization domains. The end users here are very experienced in one form of visualization, the individual slide review. In this situation, it appears crucial for the positive response to the proposed, new visualization system that it includes views and interactions that are quite close to the traditional approach. For instance, even if the 2D stack browsing was gradually abandoned, it was an intermediate step that appeared to shorten the path to the more advanced interaction.

\subsection{Domain-specific Insights}

The results described above reflect that the main pathology-specific insights enabled by the visualization revolve around the improved
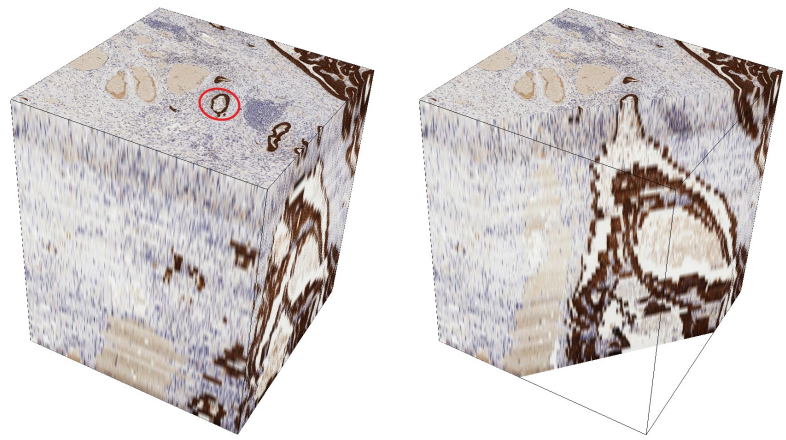

Fig. 13. Investigating a gland candidate (indicated by the red circle) in the colorectal adenocarcinoma. Clipping the image stack with the clip plane reveals that the gland candidate extends over the entire stack, thus clearly indicating a benign glandular structure and not a tumor region.

spatial understanding of the tissue at hand. Many comments to this end were made, such as "it's not easy to get a $3 D$ picture of a tumor when I look at 2D slides." and "you can get 3D [tissue samples] visualized and not just in your imagination."

Examples of specific insights were described while interacting with the datasets. For instance, for the liver case in Task 2 the pathologists developed a thorough understanding of the vessels' topology and shape. Through clipping, an entire vessel could be studied in relation to the surrounding functional liver tissue.

An illustrative example of 3D-assisted insights comes from the adenocarcinoma (Fig. 9d) in Task 3. A decisive diagnostic finding is whether the cancer is invasive or not. Regular glands can, however, in some situations be mistaken for invasive cancer in $2 \mathrm{D}$ images. The visualization on the left of Fig. 13 features an interesting oval structure, indicated by the red circle that could be a potential indication of a tumor region if it was mostly disconnected from surrounding tissue. The pathologist in question used the clip plane to reveal cross-sectional connection of the structure: a benign gland extending throughout the stack (Fig. 13, right). Thus, he concluded that it is part of the glandular structures within the polyp and, hence, no tumor region. To reach the same conclusion in clinical practice, the pathologist would have needed to request additional sections from the specimen (typically meaning a waiting time of at least one day) and then manually locating the matching position on neighboring slides, while perhaps still not being completely certain of the gland connection.

One expert pointed out that the identification of vascular invasion is a similar use case for 3D histopathology. Here, the pathologist needs to decide whether a tumor is located within the vessel. Apart from studying arteries and glands, the pathologists also mentioned isolating fistulae, an abnormal channel between two structures which are normally not connected, as a useful application. Another application area mentioned was tumor margin assessment.

The mouse embryo dataset also sparked much interest for 3D exploration. The advantage of 3D visualization to understand the extent and relations of organs and body parts including their varied normal histological appearances was apparent.

\subsection{Technical results}

We measured the performance of our system for the Barrett's esophagus dataset on an Intel Xeon at $2 \mathrm{GHz}, 6$ logical cores, $16 \mathrm{GiB}$ RAM and a NVIDIA GeForce GTX TITAN with 6 GiB VRAM. We empirically found that a brick size of $128 \times 128 \times n$ works best for the datasets at hand for both data loading and volume rendering. Tests were performed with brick dimensions ranging from 32 to 256 . The pyramidal TIFF image files were located on an SSD consuming $124 \mathrm{GiB}$ in total for this dataset with a jpeg compression ratio of 1 to 4 , but only every other LoD level. This choice was made by the institute responsible for digitizing the slide images to save both time for downsizing and tiling as well as disk space. The viewport resolution for the raycasting was set to $1,920 \times 1,080$ while the subvolume was fitted to vertically fit the screen 
Table 1. Performance measurements for Barrett's esophagus at two different levels of detail. Volume rendering was measured for two camera settings: static top-down camera (top) and a camera rotating around the subvolume (avg). The viewport resolution was 1,920 ×1,080.

\begin{tabular}{ll}
\hline $\mathbf{6 2 5}$ bricks, $\mathbf{3 2 0 0} \times \mathbf{3 2 0 0} \times \mathbf{1 0 2}$ & $\mathbf{4 . 1 7 8 ~ G i B}$ of image data \\
\hline Loading & $8.64 \mathrm{sec}$ \\
Throughput & $485 \mathrm{MiB} / \mathrm{sec}$ \\
Volume rendering & $63.4 \mathrm{fps}($ top), $49.4 \mathrm{fps}(\mathrm{avg})$ \\
with slide background hidden & $23.2 \mathrm{fps}($ top), $11.6 \mathrm{fps}(\mathrm{avg})$ \\
\hline & \\
\hline $\mathbf{4 9}$ bricks, $\mathbf{8 9 6} \times \mathbf{8 9 6} \times \mathbf{1 0 2}$ & $\mathbf{3 2 8} \mathbf{M i B}$ of image data \\
\hline Loading & $0.69 \mathrm{sec}$ \\
Throughput & $472 \mathrm{MiB} / \mathrm{sec}$ \\
Volume rendering & $64.9 \mathrm{fps}($ top), 50.2 fps (avg) \\
with slide background hidden & $35.5 \mathrm{fps}$ (top), 27.6 fps (avg) \\
\hline
\end{tabular}

and a target resolution of the volume of $1,024 \times 1,024 \times 102$ voxels. Since the dataset features only every other LoD level, we allow for oversampling of the coarser level before switching to the next effectively resulting in a subvolume resolution between $896 \times 896$ ( 7 by 7 bricks) and $3200 \times 3200$ ( 25 by 25 bricks). It is worth pointing out that loading that many bricks at once is only necessary when switching LoD levels. Regular slide panning naturally only requires loading bricks along the edges. The results are reported in Table 1.

The time it takes to load the image data from disk and placing it into the texture atlas on the GPU corresponds to a throughput of about $480 \mathrm{MiB}$. The latency is hidden by keeping the application responsive while loading bricks in the background and displaying existing data until the new data arrives.

Some of the feedback gathered concerns technical aspects of 3D histopathology in general and our tool in particular. Even with consecutive slides, the resolution in the z-direction (between slides) is much lower than within the slide. The $\mathrm{z}$-direction jaggedness becomes more apparent the smaller the region covered by the subvolume. This is an inherent property of the histopathology preparation process since the section needs to be a few micrometer thick in order to avoid tears, whereas the scan resolution typically is at submicrometer level.

\section{Conclusion}

In this paper, we have presented a novel visualization system for volumetric histopathology data in the native resolution produced by WSI scanners. The development included addressing a number of challenging demands regarding user experience and technical performance. The positive results from our user evaluation with domain experts show that we have accomplished the goal of providing a tool that opens new avenues for their work in research and the clinical setting. The evaluation also indicates that 3D histology visualization supports the expert in getting a more complete volumetric understanding of the tissue at hand in contrast to a pure mental model. For tissue structures such as vessels and glands, the utility of 3D visualization is particularly pertinent.

The traditional views and interactions in volume rendering were not suitable for the very large and dense histology data. For instance, clipping at the near plane of the view frustum caused disorientation in close-up views. The subvolume scene solution effectively addresses this issue. The set of necessary interactions for this type of data is large - camera movement, zooming, panning, and clipping/cropping - while the effects on the resulting views can be similar. However, it is difficult to avoid confusing a novice user. Our approach to tackle this challenge might be useful to other application domains as well: subvolume views with a few attached widgets, combined with separated interaction modes.

There are few development ideas we would like to pursue in future work. Introducing a special input device such as a spacemouse may further simplify the interaction, perhaps dedicating one part of the set of interactions to the device. The overall 3D histopathology workflow would benefit from doing post-processing and registration of slide images on-the-fly, reducing overhead in data storage and transfer. A wide visualization research agenda concerns ways to tackle the high density of the data. Currently, sparsity can be introduced based on color similarity. A compelling vision would be to be able to differentiate tissue components effectively, such as controlling visibility of epithelium or stroma on-the-fly, or visualizing nothing but cell nuclei in the data by applying, e.g. a variety of transfer functions or a segmentation. The solution lies in clever combinations of advances in both image analysis and visualization. Finally, slide registration errors could potentially be misleading for close-up 3D exploration. We believe that tailored uncertainty visualization techniques could effectively address this issue.

\section{ACKNOWLEDGMENTS}

The authors would like to thank Derek Magee for his input and fruitful discussions as well as the anonymous reviewers for their comments. This work was supported through grants from the Excellence Center at Linköping and Lund in Information Technology (ELLIIT), the Swedish e-Science Research Centre (SeRC), and the DigiPat project by VINNOVA grant 2014-04257.

The Barrett's histology dataset was kindly provided by Dr. Marnix Jansen, UCLH and Barts, London, UK. The liver cirrhosis data, the healthy liver data, and the mouse embryo data are courtesy of University of Leeds, UK. The image slides of the colorectal polyp were provided by Prof. Neil Shepherd of Gloucester Royal Infirmary, UK, and scanned by University of Leeds, UK.

The presented concepts have been developed and evaluated in the Inviwo framework (www. inviwo.org).

\section{References}

[1] 3D slicer. http://slicer.org . Last accessed June 2018

[2] Slideatlas. http://slideatlas.kitware.com . Last accessed June 2018.

[3] B. E. Bejnordi, M. Veta, P. J. van Diest, B. van Ginneken, N. Karssemeijer, G. Litjens, and J. A. M. van der Laak. Diagnostic assessment of deep learning algorithms for detection of lymph node metastases in women with breast cancer. JAMA, 318(22):2199-2210, 2017. doi: 10.1001/jama. 2017.14585

[4] J. Beyer, M. Hadwiger, A. Al-Awami, W.-K. Jeong, N. Kasthuri, J. W. Lichtman, and H. Pfister. Exploring the connectome: Petascale volume visualization of microscopy data streams. IEEE Computer Graphics and Applications, 33(4):50-61, 2013. doi: 10.1109/MCG.2013.55

[5] J. Beyer, M. Hadwiger, T. Möller, and L. Fritz. Smooth mixed-resolution GPU volume rendering. In IEEE/EG Symposium on Volume and PointBased Graphics, 2008. doi: 10.2312/VG/VG-PBG08/163-170

[6] J. Beyer, M. Hadwiger, and H. Pfister. State-of-the-art in GPU-based largescale volume visualization. Computer Graphics Forum, 34(8):13-37, 2015. doi: $10.1111 /$ cgf. 12605

[7] J. Chuang, D. Weiskopf, and T. Möller. Hue-preserving color blending. IEEE Transactions on Visualization and Computer Graphics, 15(6):12751282, 2009. doi: 10.1109/TVCG.2009.150

[8] J. de Ryk, E. Namati, J. M. Reinhardt, C. Piker, Y. Xu, L. Liu, E. A. Hoffman, and G. McLennan. A whole organ serial sectioning and imaging system for correlation of pathology to computer tomography. In ThreeDimensional and Multidimensional Microscopy: Image Acquisition and Processing XI, vol. 5324, p. 224, 2004. doi: 10.1117/12.529137

[9] R. A. Drebin, L. Carpenter, and P. Hanrahan. Volume rendering. Computer Graphics (Proceedings of SIGGRAPH 1988), 22(4):65-74, 1988. doi: 10. $1145 / 54852.378484$

[10] D. Ebert, T. McClanahan, P. Rheingans, and T. Yoo. Direct volume rendering from photographic data. In Eurographics / IEEE VGTC Symposium on Visualization, 2000. doi: 10.2312/VisSym/VisSym00/137-146

[11] M. Falk, I. Hotz, P. Ljung, D. Treanor, A. Ynnerman, and C. Lundström. Transfer function design toolbox for full-color volume datasets. In IEEE Pacific Visualization Symposium (PacificVis 2017), pp. 171-179, 2017. doi: 10.1109/PACIFICVIS.2017.8031591

[12] A. Fedorov, R. Beichel, J. Kalpathy-Cramer, J. Finet, J.-C. Fillion-Robin, S. Pujol, C. Bauer, D. Jennings, F. Fennessy, M. Sonka, J. Buatti, S. Aylward, J. V. Miller, S. Pieper, and R. Kikinis. 3D Slicer as an image computing platform for the quantitative imaging network. Magnetic Resonance Imaging, 30(9):1323-1341, 2012. doi: 10.1016/j.mri.2012.05. 001 
[13] M. Gargesha, M. Qutaish, D. Roy, G. Steyer, H. Bartsch, and D. L. Wilson. Enhanced volume rendering techniques for high-resolution color cryoimaging data. In SPIE Medical Imaging 2009: Biomedical Applications in Molecular, Structural, and Functional Imaging, vol. 7262, pp. 72622V72622V-7, 2009. doi: 10.1117/12.813756

[14] E. Gobbetti, F. Marton, and J. A. Iglesias Guitián. A single-pass GPU ray casting framework for interactive out-of-core rendering of massive volumetric datasets. The Visual Computer, 24(7):797-806, 2008. doi: 10. 1007/s00371-008-0261-9

[15] A. Goode, B. Gilbert, J. Harkes, D. Jukic, and M. Satyanarayanan. OpenSlide: A vendor-neutral software foundation for digital pathology. Journal of Pathology Informatics, 4(27), 2013.

[16] S. Guthe, M. Wand, J. Gonser, and W. Straßer. Interactive rendering of large volume data sets. In IEEE Visualization 2002, pp. 53-60, 2002.

[17] M. Hadwiger, J. Beyer, W. K. Jeong, and H. Pfister. Interactive volume exploration of petascale microscopy data streams using a visualizationdriven virtual memory approach. IEEE Transactions on Visualization and Computer Graphics, 18(12):2285-2294, 2012. doi: 10.1109/TVCG.2012. 240

[18] M. Isenburg, P. Lindstrom, and H. Childs. Parallel and streaming generation of ghost data for structured grids. IEEE Computer Graphics and Applications, 30(3):32-44, 2010. doi: 10.1109/MCG.2010.26

[19] A. Khan, N. Rajpoot, D. Treanor, and D. Magee. A nonlinear mapping approach to stain normalization in digital histopathology images using image-specific color deconvolution. IEEE Transactions on Biomedical Engineering, 61(6):1729-1738, 2014. 00020. doi: 10.1109/TBME.2014. 2303294

[20] J. Kniss, G. Kindlmann, and C. Hansen. Multidimensional transfer functions for interactive volume rendering. IEEE Transactions on Visualization and Computer Graphics, 8(3):270-285, 2002. doi: 10.1109/TVCG.2002. 1021579

[21] P. Ljung, J. Krüger, E. Gröller, M. Hadwiger, C. D. Hansen, and A. Ynnerman. State of the art in transfer functions for direct volume rendering. Computer Graphics Forum, 35:669-691, 2016. doi: 10.1111/cgf.12934

[22] D. Magee, Y. Song, S. Gilbert, N. Roberts, N. Wijayathunga, R. Wilcox, A. Bulpitt, and D. Treanor. Histopathology in 3D: From three-dimensional reconstruction to multi-stain and multi-modal analysis. Journal of Pathology Informatics, 6(1), 2015. doi: 10.4103/2153-3539.151890

[23] E. A. Mateos, F. Caballero-Alemán, M. Albarracín-Ferrer, F. CárcelesMoreno, R. Hernández-Gómez, S. Hernández-Kakauridze, L. HernándezSabater, I. Jiménez-Zafra, A. López-Alacid, C. Moreno-Salmerón, M. Pérez-Ramos, A. Nieto-Olivares, N. Sánchez-Campoy, I. M. GonzálezMoro, and E. Poblet. Research on devices for handling whole slide images on pathology workstations. an ergonomic outlook. Diagnostic Pathology, 2(1), 2016. doi: 10.17629/www.diagnosticpathology.eu-2016-2:232

[24] J. Molin, M. Fjeld, C. Mello-Thoms, and C. Lundström. Slide navigation patterns among pathologists with long experience of digital review. Histopathology, 67(2), 2015. doi: 10.1111/his. 12629
[25] J. Molin, C. Lundström, and M. Fjeld. A comparative study of input devices for digital slide navigation. Journal of Pathology Informatics, 6(1):7, 2015. doi: 10.4103/2153-3539.151894

[26] C. J. Morris and D. Ebert. Direct volume rendering of photographic volumes using multi-dimensional color-based transfer functions. In Joint Eurographics and IEEE TCVG Symposium on Visualisation (VisSym 2002), pp. 115-124, 2002. doi: 10.2312/VisSym/VisSym02/115-124

[27] M. Müller, I. de Sena Oliveira, S. Allner, S. Ferstl, P. Bidola, K. Mechlem, A. Fehringer, L. Hehn, M. Dierolf, K. Achterhold, B. Gleich, J. U. Hammel, H. Jahn, G. Mayer, and F. Pfeiffer. Myoanatomy of the velvet worm leg revealed by laboratory-based nanofocus X-ray source tomography. Proceedings of the National Academy of Sciences of the United States of America, 114(47):12378-12383, 2017. doi: 10.1073/pnas.1710742114

[28] M. Onozato, M. Merren, and Y. Yagi. Automated 3D-reconstruction of histological sections. American Journal of Clinical Pathology, 138(suppl 1):A311-A311, 2012. doi: 10.1093/ajcp/138.supp11.288

[29] N. Roberts, D. Magee, Y. Song, K. Brabazon, M. Shires, D. Crellin, N. M. Orsi, R. Quirke, P. Quirke, and D. Treanor. Toward routine use of 3D histopathology as a research tool. The American Journal of Pathology, 180(5): 1835-1842, 2012. doi: 10.1016/j.ajpath.2012.01.033

[30] R. A. Ruddle, R. G. Thomas, R. Randell, P. Quirke, and D. Treanor. The design and evaluation of interfaces for navigating gigapixel images in digital pathology. ACM Transactions on Computer-Human Interaction, 23(1):1-29, 2016. doi: 10.1145/2834117

[31] C. T. Rueden, J. Schindelin, M. C. Hiner, B. E. DeZonia, A. E. Walter, E. T. Arena, and K. W. Eliceiri. ImageJ2: ImageJ for the next generation of scientific image data. BMC Bioinformatics, 18(1):529, 2017. doi: 10. 1186/s12859-017-1934-z

[32] G. Stålhammar, S. Robertson, L. Wedlund, M. Lippert, M. Rantalainen, J. Bergh, and J. Hartman. Digital image analysis of Ki67 in hot spots is superior to both manual Ki67 and mitotic counts in breast cancer. Histopathology, 2018. doi: 10.1111/his. 13452

[33] N. Stathonikos, M. Veta, A. Huisman, and P. J. van Diest. Going fully digital: Perspective of a dutch academic pathology lab. Journal of Pathology Informatics, 4(1):15, 2013. doi: 10.4103/2153-3539.114206

[34] S. Thorstenson, J. Molin, and C. Lundström. Implementation of largescale routine diagnostics using whole slide imaging in sweden: Digital pathology experiences 2006-2013. Journal of Pathology Informatics, 5(14), 2014. doi: 10.4103/2153-3539.129452

[35] Y. Xu, J. G. Pickering, Z. Nong, E. Gibson, J.-M. Arpino, H. Yin, and A. D. Ward. A method for 3D histopathology reconstruction supporting mouse microvasculature analysis. PLOS ONE, 10(5):e0126817, 2015. doi: 10.1371/journal.pone.0126817 